\title{
Optimal Techniques for mRNA Extraction from Neonatal Salivary Supernatant
}

\author{
Jessica A. Dietz ${ }^{a}$ Kirby L. Johnson ${ }^{b, c}$ Heather C. Wick ${ }^{d}$ Diana W. Bianchi ${ }^{a-c}$ \\ Jill L. Maron ${ }^{\mathrm{a}, \mathrm{c}}$ \\ Divisions of a Newborn Medicine and ${ }^{b}$ Genetics, Department of Pediatrics, The Floating Hospital for Children at \\ Tufts Medical Center, ${ }^{C}$ Mother Infant Research Institute at Tufts Medical Center, and ${ }^{\mathrm{d}}$ Department of Computer \\ Science at Tufts University, Medford, Mass., USA
}

\section{Key Words}

Neonate $\cdot$ Saliva $\cdot$ Transcriptome $\cdot$ Supernatant

\begin{abstract}
Background: Gene expression profiling of the salivary supernatant is emerging as a new and important source of real-time, systemic, biological information. However, existing technologies prevent RNA extraction of small quantities found in neonatal salivary supernatant. Objective: The aim of this study was to develop techniques to enhance extraction of cell-free RNA from neonatal salivary supernatant. Methods: Two saliva samples (10-100 $\mu \mathrm{l})$ were serially collected from newborns (36-41 weeks' gestation) $(n=13)$ and stabilized. Total RNA was extracted from salivary supernatant with the use of two modified extraction techniques: Qiagen RNAprotect ${ }^{\circledR}$ Saliva Mini Kit (method 1) and the QIAamp Viral RNA Mini Kit (method 2). Quantitative RT-PCR amplification for GAPDH was performed on extracted salivary samples. Statistical analyses were performed on mean threshold cycle (Ct) levels to compare RNA yield from each protocol. Paired microarray analyses were made between neonatal whole saliva and supernatant $(n=3)$ to discern
\end{abstract}

gene expression differences between these biolayers. $\boldsymbol{R} \boldsymbol{e}$ sults: mRNA was successfully extracted and amplified from all salivary supernatant samples. Extraction with method 2 yielded more RNA than with method $1(p=0.008)$. There was a 7.5\% discordance between paired gene expression analyses for whole saliva and supernatant. Genes that were statistically significantly upregulated in supernatant highlighted 16 distinct biological functions not seen in whole saliva. Conversely, only two biological functions were unique to whole saliva. Conclusion: Neonatal cell-free salivary supernatant mRNA may be readily extracted and utilized on downstream applications. These technical enhancements allow for further exploration of the diagnostic potential of the neonatal salivary transcriptome.

Copyright $\odot 2011$ S. Karger AG, Basel

\section{Introduction}

The development and utilization of noninvasive techniques to identify informative biomarkers in the neonate holds great promise for improving clinical care [1]. Saliva is rapidly emerging as a noninvasive source of important

\section{KARGER}

(C) 2011 S. Karger AG, Basel

Fax +41613061234 E-Mail karger@karger.ch www.karger.com www.karger.com/neo
Assist. Prof. Jill L. Maron, MD, MPH

Mother Infant Research Institute at Tufts Medical Center

800 Washington Street, Box 44

Boston, MA 02111 (USA)

Tel. +1 617636 0766, E-Mail jmaron@ @uftsmedicalcenter.org 
biological information $[2,3]$. Saliva is formed following filtration of whole blood in the salivary glands. It is composed of water, electrolytes, proteins, microorganisms, and genetic material, including both cellular and cell-free deoxyribonucleic acid (DNA) and ribonucleic acid (RNA). As a product of whole blood filtration, cell-free genetic material in saliva originates from multiple sources within the body, while the cellular genetic material is derived almost exclusively from the oral mucosa. Therefore, saliva, particularly the cell-free salivary supernatant, is an ideal body fluid to noninvasively monitor systemic organ development, physiology and disease sequelae in the neonatal population [4]. While there has been extensive research on the diagnostic potential of saliva in the dental field $[5,6]$, the clinical research applications of salivary analyses in neonatology have been underappreciated.

Recent technical advances have made the stabilization of salivary RNA possible [5]. This is a significant achievement, as ubiquitous RNases in the mouth have the potential to rapidly degrade RNA transcripts within minutes of salivary acquisition. By stabilizing RNA transcripts in saliva, gene expression analysis, also known as 'transcriptomics', can now provide the neonatal researcher with the opportunity to monitor real-time developmental and diagnostic information about the preterm neonate. However, unlike in an adult or older child, neonates cannot generate saliva on demand. In addition, the limited neonatal salivary sample volume (approximately 10 up to 100 $\mu \mathrm{l} /$ per sample) prevents analysis of the cell-free supernatant component.

Our group was one of the first to publish on the salivary transcriptome of the premature neonate [7]. This initial study was conducted on whole salivary samples. While this research demonstrated that whole saliva was a source of mRNA in the neonate, it contrasted with adult literature that has largely focused on the cell-free supernatant in order to reduce the genomic contribution from the oral mucosa $[6,8]$. In addition, abundant gene transcripts from cellular sources impact upon hybridization rates in microarray experiments. The most common example of this is the detection of hemoglobin genes in whole blood [9]. When attempting to analyze thousands of genes from quantitatively limited starting sources (as in neonatal salivary microarray experiments), this interference can result in lower gene detection rates for rare sequences, greater variance, and decreased sensitivity [10].

We wished to use the cell-free supernatant to explore systemic pathophysiology in the neonate. To do this, we had to optimize acquisition techniques and protocols to utilize this important source of information in neonates without the interference of oral cellular genetic material. Here, we describe and compare two enhanced approaches for obtaining neonatal cell-free salivary supernatant RNA, and describe optimized acquisition and processing protocols to maximize RNA yield from extremely limited volumes of neonatal saliva. In addition, we perform paired microarray analyses between whole saliva and cell-free salivary supernatant to better elucidate gene expression differences between these biofluids and highlight unique biological pathways present in each salivary component.

\section{Materials and Methods}

This study was approved by the Tufts Medical Center Institutional Review Board. Informed parental consent was obtained for all neonates ( $n=16$; gestational ages: $365 / 7$ to 41 weeks' gestation) enrolled in this study. Infants were only selected for participation if they were healthy, without known anomalies or genetic syndromes, and were being cared for in the normal newborn nursery at Tufts Medical Center. Samples were collected only once from each infant on day 0 to 4 of life. Saliva samples were collected with previously developed techniques [7] (fig. 1). Briefly, samples were collected with a 1-ml syringe with plunger and endcaps removed. The syringe was attached to low wall suction $(<20 \mathrm{~mm} \mathrm{Hg})$ to ensure that saliva remained in the syringe and did not enter into the tubing or suction trap. Saliva was suctioned from under the neonates' tongues and in their gingival crevices, where it is known to pool. Suctioning occurred for approximately $10-15 \mathrm{~s}$. The syringe was removed from suction and the plunger was returned where it was used to transfer the saliva directly into the RNAprotect ${ }^{\circledR} \mathrm{Sa}$ liva reagent. Samples were aspirated three to five times to ensure all saliva was removed from the syringe. Samples were immediately vortexed, placed on ice and brought back to the laboratory for further processing. Neonates had not eaten within $1 \mathrm{~h}$ of sample collection to limit breast milk or formula contamination.

\section{Acquisition and Initial Processing}

Of the 16 enrolled neonates, 13 had two consecutive salivary samples collected within $60 \mathrm{~s}$. The order of the processing of each salivary sample was randomized so as not to skew the data. All samples were immediately placed in $1 \mathrm{ml}$ of RNAprotect Saliva (Qiagen GMBH Hilden, Germany). RNAprotect Saliva deactivates ubiquitous RNases present in the mouth and inhibits uncontrolled growth of oral bacteria, both of which can interfere with RNA extraction. Therefore, it is essential that saliva be placed into this reagent immediately after acquisition to ensure reliable gene expression results. All samples were centrifuged at 5,500 rpm $(2,469 \mathrm{~g})$ for $15 \mathrm{~min}$ at $4^{\circ} \mathrm{C}$ in order to separate cellular and cell-free components. Salivary supernatant was removed, placed into separate Eppendorf tubes, and left at $4^{\circ} \mathrm{C}$ for a minimum of $48 \mathrm{~h}$ prior to RNA extraction. Although manufacturer's instructions recommend a minimum of $24 \mathrm{~h}$ prior to RNA extraction, our laboratory has found that $48 \mathrm{~h}$ improves both qualitative and quantitative yield. 
Fig. 1. Schematic of neonatal salivary acquisition. Step 1: 1-ml syringe is first modified by removing the endcaps; step 2: the plunger is removed, saved and set aside; step 3: the modified syringe is attached to low wall suction and salivary sample is gently collected from the neonate's oropharynx; step 4: the sample is immediately placed into $1 \mathrm{ml}$ of RNAProtect Saliva Solution; step 5: the sample is vortexed for $20 \mathrm{~s}$; step 6: the sample is placed immediately on ice; step 7: the sample is brought back to the laboratory for further processing.

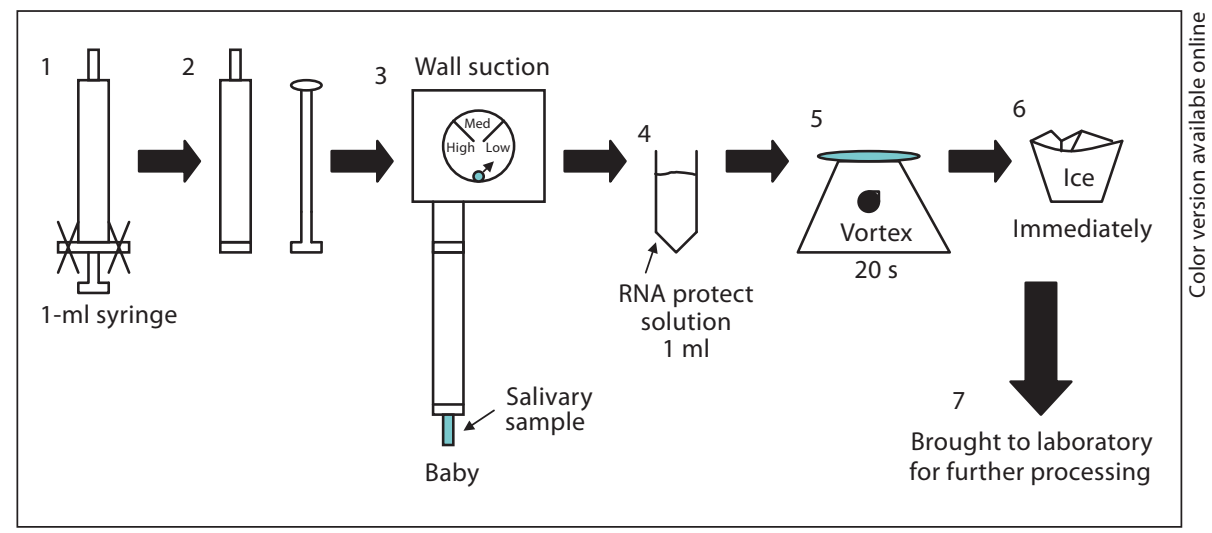

\section{RNA Extraction}

The RNA was extracted using two different methods. Total RNA using method 1 was extracted with the use of the Qiagen RNAprotect Saliva Mini Kit (Qiagen) as per the manufacturer's protocol. This protocol was designed to extract salivary RNA directly from whole saliva. However, we also utilized this protocol to determine if RNA could be extracted from one of the cell-free supernatant components. The final elution volume was $14 \mu$ l. Total RNA using method 2 was extracted using a modified protocol of the QIAamp Viral RNA Mini Kit (Qiagen) (table 1), a protocol originally developed for the extraction of RNA from salivary supernatant. The final elution volume for these samples was $30 \mu \mathrm{l}$. On column DNase digestion was carried out on all samples to eliminate DNA contamination. Extracted total RNA was stored at $-80^{\circ} \mathrm{C}$ pending further analysis.

\section{Quantitative Reverse Transcription-Polymerase Chain}

\section{Reaction}

One-step quantitative reverse transcription-polymerase chain reaction (qRT-PCR) amplification for glyceraldehyde phosphate dehydrogenase $(G A P D H)$ was performed on the salivary samples processed by each method. Samples were run in duplicate with negative controls on the Perkin-Elmer Applied Biosystems 7900 Sequence Detector with the TaqMan ${ }^{\circledR}$ One-Step RT-PCR Master Mix Reagents Kit (Applied Biosystems, Foster City, Calif., USA) as previously described [11]. Amplification primer and probe sequences for GAPDH (Applied Biosystems) have been previously reported [12].

The threshold cycle $(\mathrm{Ct})$ values of each sample were used in our calculations. Ct refers to the amplification cycle at which one can discriminate between true gene amplification versus background fluorescence. Thus, transcripts with the highest concentration will amplify early and have a lower Ct value. Ct values with method 2 were adjusted for increased elution volume as follows: based upon a 2.14-fold greater elution volume in these samples (derived by dividing the elution volume of method $2[30 \mu \mathrm{l}]$ by the elution volume of method $1[14 \mu \mathrm{l}]$ ), Ct values from method 2 were adjusted with the following equation: $2^{\mathrm{x}}=2.14$. The Ct level was subsequently adjusted by the resulting numerical value, 1.099. Mean Ct values were used in the statistical analyses.
Gene Expression Microarray Analyses: Whole Salivary versus Salivary Supernatant

Salivary samples from the remaining 3 term neonates enrolled in this study were used to compare gene expression differences between whole saliva and the cell-free supernatant. Consecutively collected samples were randomized to either whole saliva, extracted with the use of the Qiagen RNAprotect Saliva Mini Kit (Qiagen), as per the manufacturer's protocol, or cell-free supernatant, extracted with the modified protocol of the QIAamp Viral RNA Mini Kit (Qiagen). Total RNA was amplified from all samples using the Ovation Pico WTA System (NuGEN ${ }^{\circledR}$ Technologies, San Carlos, Calif., USA). The quantity and quality of the amplified cDNA was assessed with an Agilent Bioanalyzer 2100 (Agilent Technologies, Santa Clara, Calif., USA) before fragmentation and biotinylation with the Encore Biotin Module (NuGEN Technologies). For all samples, a standard $5 \mu \mathrm{g}$ of amplified and labeled cDNA was hybridized onto an Affymetrix GeneChip Human Genome U133a 2.0 Plus microarray (Affymetrix, Santa Clara, Calif., USA). Following hybridization, each array was washed and stained in the GeneChip Fluidics Station 400 (Affymetrix) and scanned with the GeneArray Scanner (Affymetrix).

\section{Statistical Analyses}

Statistical analyses for RNA extraction techniques and qRTPCR data were performed with SPSS 11.5 software (IBM, Somers, N.Y., USA). Independent $t$ tests were performed between mean Ct levels from each protocol to compare RNA yield. Statistical significance was set at a threshold level of $\mathrm{p}<0.05$.

Microarray data underwent a quantile normalization with ideal mismatch background correction and Tukey Biweight summarization. Following normalization, paired t tests were performed between whole saliva and supernatant gene expression profiles to identify statistically significantly differentially expressed genes. All $p$ values were adjusted for a false discovery rate with the Benjamini-Hochberg procedure [13]. Statistical significance was set at a threshold level of an adjusted p value of $<0.05$. Statistically significantly differentially expressed genes for each salivary biolayer were further analyzed with the use of the Ingenuity ${ }^{\circledR}$ Pathway Analysis (IPA) system. IPA is a commercially available software analysis program that allows the researcher to identify and interpret relevant biological information from gene lists [14]. 


\section{Results}

\section{Comparison of Extraction Methods}

The quantity of salivary volumes collected ranged from approximately $10-100 \mu l$. Total RNA was successfully extracted from all salivary supernatant samples. Mean Ct level from method 1 was 31.8 (SD 2.57; range 25.6-35.6); adjusted mean Ct level from method 2 was 29.4 (SD 1.57; range 28.0-32.9). The modified protocol and QIAamp Viral RNA Mini Kit (method 2) yielded more RNA with less variation compared to the RNAprotect Saliva Mini Kit (method 1) (t test $p=0.008)$. Paired whole saliva and cell-free supernatant comparisons revealed mean Ct levels of GAPDH in whole saliva of 27.2 (SD 0.86; range 26.35-28.3) and mean Ct levels in the cellfree supernatant component of 29.9 (SD 2.01; range 27.832.25) ( $\mathrm{t}$ test $\mathrm{p}=0.034)$.

\section{Comparison of Biolayers}

There was a $92.5 \%$ gene expression concordance between paired whole saliva and cell-free salivary supernatant samples, with only $7.5 \%$ of the genes statistically significantly differentially expressed. There were 1,474 genes that had statistically significantly higher gene expression in the cell-free supernatant layer, while 1,485 genes had statistically significantly higher gene expression in whole saliva. Following analyses of each respective gene list with IPA, we were able to identify 16 unique biological systems in the cell-free supernatant layer that were not detectable in whole saliva. These systems included 'tissue morphology', 'organismal development', 'respiratory system development and function', and 'lymphoid tissue structure and development'. Conversely, only two biological systems, 'free radical scavenging' and 'protein degradation', were solely represented in whole saliva (table 2).

\section{Discussion}

Neonatal salivary transcriptomic analyses represent an innovative and noninvasive means to monitor the newborn population. The aim of this study was to develop techniques to extract cell-free salivary supernatant RNA in order to limit the contribution of cellular RNA in neonatal samples and enhance the detection of systemic gene transcripts on microarray hybridizations. Our results reveal that with the optimized techniques developed here, mRNA can be detected, amplified, and analyzed in the supernatant neonatal salivary compo-
Table 1. Modified salivary supernatant extraction protocol (QIAamp Viral RNA Mini Kit)

Collection

1 Centrifuge samples after initial stabilization in $1 \mathrm{ml}$ RNAprotect Saliva at 5,500 rpm $(2,469 \mathrm{~g})$ for $15 \mathrm{~min}$ at $4^{\circ} \mathrm{C}$

2 Aliquot supernatant component into new 1.5-ml Eppendorf tube and store at $4^{\circ} \mathrm{C}$ for a minimum of $48 \mathrm{~h}$ up to 4 weeks

Reagents and equipment required

1 QIAamp Viral RNA Mini Kit

(QIAGEN catalog No.: 52904)

2 Qiagen RNAProtect Saliva Mini Kit

(QIAGEN catalog No.: 74324)

3 RNase-Free DNase Set

(QIAGEN catalog No.: 79254)

$4 \quad 100 \%$ molecular biology grade ethanol

5 Microcentrifuge

6 Vortex

RNA extraction protocol

1 Retrieve stabilized salivary supernatant samples from $4^{\circ} \mathrm{C}$

2 Mix $140 \mu \mathrm{l}$ of stabilized salivary supernatant with $560 \mu \mathrm{l}$ buffer AVL (a guanidine hydrochloride-based lysis buffer in Viral RNA Mini Kit) in a 2-ml tube

(note: Due to the increased starting volume of stabilized salivary supernatant $(\sim 500 \mu \mathrm{l})$, a minimum of 4 tubes per sample will be required)

3 Allow the salivary supernatant/buffer AVL mixture to incubate at room temperature for $10 \mathrm{~min}$

4 Vortex for $5 \mathrm{~s}$ to $\mathrm{mix}$

5 Add $560 \mu \mathrm{l}$ of $100 \%$ molecular biology grade ethanol to each tube

6 Pipette $630 \mu$ l of the sample mixture onto the spin column and spin at $8,000 \mathrm{rpm}(11,029 \mathrm{~g})$ for $1 \mathrm{~min}$; discard collection tube; repeat this step until all of sample volume has been loaded onto the column

(note: due to the increased volume of reagents used this will take up to 9 centrifugation steps to load an entire sample's mixture onto one column)

7 Add $500 \mu$ l buffer AW1 (a wash buffer in Viral RNA Mini Kit) to column and spin at $8,000 \mathrm{rpm}(11,029 \mathrm{~g})$ for $1 \mathrm{~min}$; discard collection tube

8 DNase incubation: mix $10 \mu \mathrm{l}$ DNase I stock solution with $70 \mu \mathrm{l}$ buffer RDD (DNAse buffer) for each sample; pipette $80 \mu \mathrm{l}$ of solution onto column and incubate on bench for $15 \mathrm{~min}$ at room temperature

9 Add $500 \mu$ l buffer AW1 (a wash buffer in Viral RNA Mini $\mathrm{Kit})$ to the column and spin at $8,000 \mathrm{rpm}(11,029 \mathrm{~g})$ for 1 min; discard collection tube

10 Add $500 \mu$ l buffer AW2 (a wash buffer in Viral RNA Mini Kit) and spin at full speed for $3 \mathrm{~min}$; discard collection tube

11 Spin column at full speed for $2 \mathrm{~min}$

12 Add $30 \mu$ l of RNase-free water to the column and spin at $9,000 \mathrm{rpm}(12,407 \mathrm{~g})$ for 2 min to elute RNA

13 Extracted RNA may be placed at $-20^{\circ} \mathrm{C}$ for short-term use or at $-80^{\circ} \mathrm{C}$ for long-term storage 
nent. Supernatant extracted with the use of method 2, a modified protocol of the QIAamp Viral RNA Mini Kit, yielded more RNA than supernatant extracted with method 1, the RNAprotect Saliva Mini Kit. However, $G A P D H$ was detected in all samples with the use of either protocol. These results are encouraging and clearly demonstrate the success of the enhanced RNA isolation protocols.

A caveat to the use of the QIAamp Viral RNA Mini $\mathrm{Kit}$, as described here, is that it requires RNAprotect Saliva reagent for stabilization of salivary supernatant. Currently, RNAprotect Saliva is only available from Qiagen with the purchase of the RNAprotect Saliva Mini Kit (USD 11.30/sample). Thus, both kits would need to be purchased from Qiagen (combined USD 15.00/sample) to utilize this modified hybrid protocol. We therefore suggest that the investigator consider cost when choosing between protocols, as both yield results.

Overall, there is considerable similarity between the gene expression profiles of whole saliva and the cell-free salivary supernatant ( $92.5 \%$ concordance). This is reassuring, as significant expression differences would suggest qualitative differences between RNA extracted from each respective sample source. However, despite the similarity between the gene expression profiles, we did identify a subset of genes that were differentially expressed in each biolayer. This finding was expected. It is well known that hybridization rates in microarray studies can be impacted by abundant gene transcripts in a given sample. Similarly, we hypothesized that abundant cellular material in whole saliva may limit the detection of systemic organ development on a microarray platform. Our results validate this hypothesis and suggest that extensive systemic organ development may be more readily detected in the supernatant layer. However, we caution against the preferential use of only the neonatal salivary supernatant layer. Gene expression analyses of both salivary supernatant and whole saliva provide a large amount of real-time neonatal developmental information. Therefore, we suggest that investigators choose which biolayer best meets the needs of their study design and consider cost, processing procedures, and data interpretation when choosing a salivary source. The techniques for salivary supernatant RNA extraction described here provide the researcher with the necessary tools to make such a decision.

A potential limitation to this study is that the oral fluid collected from the neonates may not be solely salivary in origin. Residual amniotic fluid, as well as gastric and tracheal secretions may contribute to the RNA yield in
Table 2. Statistically significantly over-represented biological pathways in salivary

\begin{tabular}{ll}
\hline Biological function & p value \\
\hline Supernatant & \\
Psychological disorders & $5.05 \mathrm{E}-04-4.49 \mathrm{E}-02$ \\
Cardiovascular disease & $8.34 \mathrm{E}-04-4.75 \mathrm{E}-02$ \\
Renal and urological disease & $8.77 \mathrm{E}-04-4.60 \mathrm{E}-02$ \\
Developmental disorder & $8.74 \mathrm{E}-04-4.01 \mathrm{E}-02$ \\
Tissue morphology & $1.55 \mathrm{E}-03-2.99 \mathrm{E}-02$ \\
Organismal development & $3.59 \mathrm{E}-03-4.74 \mathrm{E}-02$ \\
Dermatological diseases and conditions & $3.71 \mathrm{E}-03$ \\
Amino acid metabolism & $5.51 \mathrm{E}-03-4.74 \mathrm{E}-02$ \\
Organ morphology & $5.50 \mathrm{E}-03-1.52 \mathrm{E}-02$ \\
Ophthalmic disease & $1.19 \mathrm{E}-02-3.90 \mathrm{E}-02$ \\
Organismal functions & $1.57 \mathrm{E}-02$ \\
Lymphoid tissue structure and development & $1.72 \mathrm{E}-02-2.99 \mathrm{E}-02$ \\
Auditory disease & $2.99 \mathrm{E}-02$ \\
RNA damage and repair & $4.01 \mathrm{E}-02$ \\
Antimicrobial response & $4.74 \mathrm{E}-02$ \\
Respiratory system development and function & $4.74 \mathrm{E}-02$
\end{tabular}

\begin{tabular}{ll}
\hline Whole saliva & \\
Free radical scavenging & $6.03 \mathrm{E}-03$ \\
Protein degradation & $1.79 \mathrm{E}-02$ \\
\hline
\end{tabular}

this study. While we cannot be certain of the purity of our samples, the material collected nevertheless represents an easily obtainable biofluid that may be used to identify potential diagnostic biomarkers in the neonatal population.

Unlike adult patients in whom salivary transcriptomes are currently being utilized to predict and diagnose disease outcomes that may occur over months to years, serious neonatal disease with the potential for lifelong morbidity occurs within hours to weeks of birth. Developing noninvasive techniques to monitor gene expression changes in relation to the onset of disease holds significant potential for improving clinical care of the neonate. While future research is needed to further elucidate the source of gene transcripts in all components of neonatal saliva, we have clearly demonstrated that RNA from the cell-free salivary supernatant component can be successfully isolated and utilized on multiple downstream platforms. These enhancements provide the investigator with the tools necessary to further explore the diagnostic potential of the neonatal salivary transcriptome. 


\section{Acknowledgments}

We would like to thank the families who graciously participated in this research, as well as the nurses and staff in the Mother Infant Unit at the Floating Hospital for Children at Tufts Medical Center. Funding for this research was provided by NICHD K08HD059819-02 and The Charles H. Hood Foundation Award to J.L.M.

\section{Disclosure Statement}

Drs. Maron, Johnson and Bianchi have a pending patent application, PCT/US2009/42626, entitled: Neonatal Salivary Genomics.

\section{References}

-1 Haak PT, Busik JV, Kort EJ, Tikonenko M, Paneth N, Resau JH: Archived unfrozen neonatal blood spots are amenable to quantitative gene expression analysis. Neonatology 2009;95:210-216.

2 Zhang L, Ziao H, Wong DT: Salivary biomarkers for clinical applications. Mol Diagn Ther 2009; 13:245-259.

>3 Zhang L, Farrell JJ, Zhou H, Elashoff D, Akin D, Park NH, Chia D, Wong DT: Salivary transcriptomic biomarkers for detection of resectable pancreatic cancer. Gastroenterology 2010;138:949-957.

4 Wong DT: Salivary diagnostics: amazing as it might seem, doctors can detect and monitor diseases using molecules found in a sample of spit. Am Sci 2008;96:37-43.

5 Segal A, Wong DT: Salivary diagnostics: enhancing disease detection and making medicine better. Eur J Dent Educ 2008;2(suppl $1): 22-29$.
6 Li Y, Zhou X, St. John MAR, Wong DTW: RNA profiling of cell-free saliva using microarray technology. J Dent Res 2004;83: 199-203.

7 Maron JL, Johnson KJ, Rocke DM, Cohen MG, Liley AJ, Bianchi DW: Neonatal salivary analysis reveals global developmental gene expression changes in the premature neonate. Clin Chem 2010;56:409-416.

8 Park NJ, Zhou X, Yu T, Brinkman BM, Zimmermann, BG, Palanisamy V, Wong DT: Characterization of salivary RNA by $\mathrm{CDNA}$ library analysis. Arch Oral Biol 2006;52:3035.

$\checkmark 9$ Wright C, Bergstrom D, Dai H, Marton M, Morris M, Tokiwa G, Wang Y, Fare T: Characterization of globin RNA interference in gene expression profiling of whole blood samples. Clin Chem 2008;54:396-405.

10 Tian Z, Palmer N, Schmid P, Yao H, Galdzicki M, Berger B, Wu E, Kohane IS: A practical platform for blood biomarker study by using global gene expression profiling of peripheral whole blood. PloS One 2009;4:e5157.
11 Livak KJ: Allelic discrimination using fluorogenic probes and the $5^{\prime}$ nuclease assay. Genet Anal 1999;14:143-149.

>12 Ng EK, Tsui NB, Lam NY, Chiu RW, Yu SC, Wong SC, Lo ES, Rainer TH, Johnson PJ, Lo YM: Presence of filterable and nonfilterable mRNA in the plasma of cancer patients and healthy individuals. Clin Chem 2002;48: 1212-1217.

13 Benjamini Y, Hochberg Y: Controlling the false discovery rate: a practical and powerful approach to multiple testing. J R Stat Soc Ser B 1995;57:289-300

14 Jiménez-Marín Á, Collado-Romero M, Ramirez-Boo M, Arce C, Garrido JJ: Biological pathway analysis by ArrayUnlock and Ingenuity Pathway Analysis. BMC Proc 2009;3(suppl 4):S6 